\title{
Neural Classifiers of Vibroacoustic Signals in Implementation on Programmable Devices (FPGA) - Comparison
}

\author{
D. DĄBROWSKi* And W. CiOCH
}

Department of Mechanics and Vibroacoustic, University of Science and Technology

\author{
al. A. Mickiewicza 30, 30-059 Kraków, Poland
}

\begin{abstract}
The research includes comparative analysis of the effect of the recording of weight vectors and input data of selected neural classifiers with fixed-point numbers. Research has been conducted due to insufficient literature on the influence of such a recording on the correct classification of vibroacoustic signals by neural networks. This current issue was brought up in authors' earlier researches, concerning realization of neural classifiers on programmable logical devices field programmable gate array, with the application of fixed-point processor. During the analysis, three types of neural classifiers were compared in the tests: classifier based on neural network length vector quantization, classifier using radial neural networks - radial basis functions, and third — counter propagation neural network. The problem stated was to recognize technical state of gear transmission DMA-1 in variable operating conditions. Vectors, based on estimates derived from processed vibroacoustic signals were used as teaching material.
\end{abstract}

PACS: $45.80 .+\mathrm{r}, 46.40 .-\mathrm{f}$

\section{Introduction}

Artificial neural networks are widely applied as classifiers in a diagnosis process. They enable the approximation of transforming multidimensional space of symptoms into classes of technical state of the examined object $[1,2]$. Local or global transformation of multidimensional space into solution space proves to be extremely difficult by means of mathematical formulae [3]. Artificial neural networks also have auto-association feature, i.e. they enable associating an image with pre-remembered pattern [4]. It is required to specify the symtoms indicating inoperability states [1] for neural classifiers to enable the identification of the state of an object. In this research estimates were built based on frequency analysis of residual processes in operating machinery. The analysis of vibroacoustic signals can be effectively assisted by hardware-software solutions $[5,6]$. Programmable diagnostic device - PUD (in Polish: programowalne urządzenie diagnostyczne) combines the advantages of hardware signal processing with computing carried out by software. Neural classifier works on fixed-point processor configured on progem logic of field programmable gate array (FPGA).

\section{Research problem}

Neural classifier was built for the purpose of recognizing the technical state of gear transmission DMG-1. Gears are widely applied in power transmission systems. They can often be weak points in kinematic chain of a machine. Gear transmission diagnosis is especially purposeful owing to easy failure identification [7-10]. Re-

\footnotetext{
* corresponding author; e-mail: dabrows@agh.edu.pl
}

search was executed on demonstrative DMG-1 gear transmission, which allowed to introduce changes in alignment, loading and rotation speed.

Signals for two classes of technical state were used in the tests, indicating alignment state and misalignment. Experiments were carried out for two rotation speeds: $900 \mathrm{rpm}$ and $1200 \mathrm{rpm}$ as well as for two states of loading: no loading and $2 \mathrm{MPa}$ loading. The vector of technical state is shown in Table I; it has been created based on symptoms associated with rotation frequency and its amplitude harmonic, as well as the ratio of second harmonic over amplitude of rotation frequency.

TABLE I

Vector of technical state of demonstrative DMG-1 gear transmission.

\begin{tabular}{c|l}
\hline \hline Estimate & \multicolumn{1}{c}{ Technical state vector } \\
\hline I & $\begin{array}{l}\text { amplitude of rotation frequency } \\
\text { amplitude of second harmonic of rotation } \\
\text { frequency } \\
\text { ratio of second-harmonic amplitude to am- } \\
\text { plitude of rotation frequency }\end{array}$ \\
III
\end{tabular}

\section{Architecture and principle of neural classifiers tested}

\subsection{Counter propagation neural network}

Counter propagation $(\mathrm{CP})$ neural network is a compilation of the Kohonen and Grossenberg architecture (Fig. 1). Simple principle of operation of the network facilitates the learning process. Moreover, possibilities of preliminary adaptative classification into stimuli and 


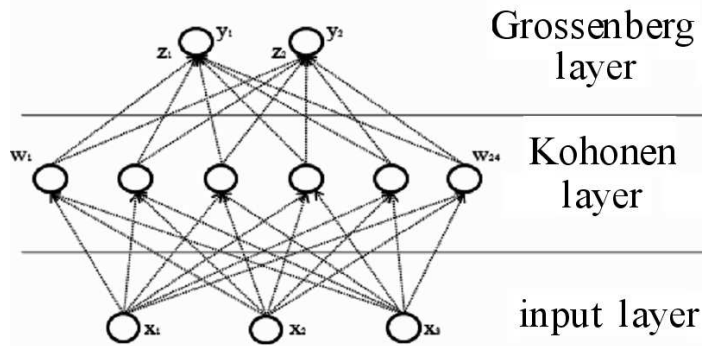

Fig. 1. Architecture of $\mathrm{CP}$ artificial neural network.

additional learning abilities prove that this type of neural networks can be applied in a great variety of devices $[12,13]$.

Learning of CP neural network is a two-stage process: in the first, the Kohonen layer is self-organized (Eq. (2)), and in the second, the Grossenberg layer is learned following the Widrow-Hoff learning rule (Eq. (4)):

$$
\begin{aligned}
& w_{m}^{t} x=\max \left(w_{i}^{t} x\right), \quad i=1, \ldots, n, \\
& w_{m}^{k+1}=w_{m}^{k}+\alpha\left(x-w_{m}^{k}\right), \\
& w_{i}^{k+1}=w_{i}^{k}, \quad i \neq m, \\
& z_{i j}^{k+1}=z_{i j}^{k}+\beta\left(y_{i}-d_{i}\right) t_{i}, \\
& t_{i}=\left\|w_{i}-x_{i}\right\|,
\end{aligned}
$$

where $w$ - weight vector, $x$ - input vector, $y$ - output vector, $d$ - learning vector, $z$ - output-layer weight vector, $k$ - time period, $m-$ number of winning neuron, $n$ - number of neurons.

Counter propagation neural networks can generalize and associate information delivered to them. They do not require extensive computations, which makes them suitable for common applications $[4,14]$.

\subsection{Length vector quantization neural network}

In this research a model of length vector quantization (LVQ) neural network was used. This model of neural network was introduced by Kohonen [13]. The LVQ artificial neural network is composed of three layers. First layer is the input layer. The second one is the Kohonen-type self-organizing layer - it classifies the input vectors into categories detected during the grouping process of the teaching set.

During the learning process, as a result of competition, the Kohonen layer is being self-organized according to Kohonen's principle (Eq. (1)). This process involves adjustment of weights of the vector that is closest to the teaching vector. In LVQ network the neurons of the Kohonen layer are ascribed to individual classes, so that the equal number of neurons are ascribed to a specific class (Fig. 2). The purpose of the exit layer is assigning the input vector to one of several classes $[3,4]$ :

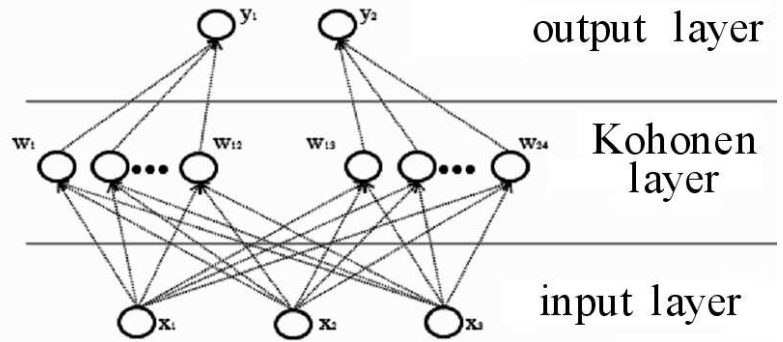

Fig. 2. Architecture of LVQ artificial neural network.

$$
\begin{aligned}
& w_{m}^{t} x=\max \left(w_{i}^{t} x\right), \quad i=1, \ldots, n, \\
& w_{m}^{k+1}=w_{m}^{k} \pm \alpha\left(x-w_{m}^{k}\right), \\
& w_{i}^{k+1}=w_{i}^{k}, \quad i \neq m,
\end{aligned}
$$

where $w$ - weight vector, $x$ - input vector, $y$ - output vector, $k$ - time period, $\mathrm{m}$ - winning neuron number, $n$ - number of neurons.

The vector quantization network is an example of strain network, which means that the individual neurons in a self-organizing layer are assigned to individual classes by a designer.

\subsection{Radial neural networks}

Radial basis function (RBF) neural networks consist of three layers. Input layer does not affect signal that is fed into the network. Hidden layer is composed of neurons which carry out function that is changing radially around the defined center, for the network discussed it would be Gaussian function.

Third layer of neural classifier is linear, enabling transforming of the whole multidimensional space, through superposition of signals from the hidden layer and suitable weight scaling. Figure 3 shows architecture of RBF neural network.

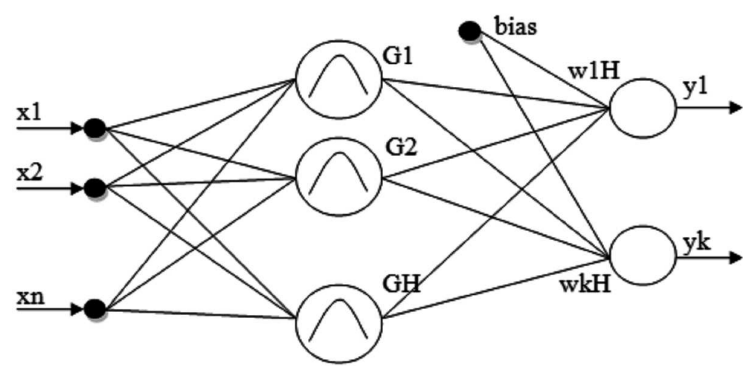

Fig. 3. Architecture of RBF artificial neural network. $x_{n}$ - input signal, $y_{n}$ - output signal, $G_{H}$ - neurons of radial base functions, $w_{k H}$ - weights of output layer.

Neural classifier based on RBF neural network has been taught in two stages. In the first stage the arrangement of radial-function centres were selected, using $K$ -means algorithm (Eq. (10)). In the second stage values of weights of output layer were specified by means of the Widrow-Hoff principle (Eq. (4)): 


$$
\begin{aligned}
& G\left(x ; c, \sigma^{2}\right)=\exp \left(-\frac{\|x-c\|^{2}}{2 \sigma^{2}}\right), \\
& E=\sum_{d=1}^{H} \sum_{r \in D_{s}}^{D}\left\|x^{d}-c_{d}\right\|^{2},
\end{aligned}
$$

where $x$ - input variable, $c$ - radial function centre, $\sigma^{2}$ - parameter of function width, $x^{d}$ - learning vectors, $H$ - number of centres, $D$ - number of learning vectors located near $d$-center.

Neural classifier based on radial neural networks does not work as classifier with neural networks with sigmoid activation function, local approximation of multidimensional space transformation into the space of fewer dimensions [2].

\section{Comparing of neural classifiers}

In the research selected neural classifiers were compared by means of fixed-point Micro Blaze processor [16]. Architecture of CP and LVQ neural network is as follows: first layer is composed of three neurons, hidden layer of twenty-four neurons and output layer composed of just two neurons. Such an architecture choice was forced by input-vector length, amount of teaching data, as well as the number of classes to recognize. Radial neural network differs from beforementioned networks in terms of number of neurons in hidden layer, for which eight neurons are assigned due to the need to split data into clusters. Architecture of the classifiers is shown in Table II.

TABLE II

Number of neurons in layers of neural networks.

\begin{tabular}{c|c|c|c}
\hline \hline Number of neurons & CP & LVQ & Radial net \\
\hline layer 1 & 3 & 3 & 3 \\
layer 2 & 24 & 24 & 8 \\
layer 3 & 2 & 2 & 2
\end{tabular}

Analysis involved verification of network classification correctness for teaching and testing data, where input vectors and weights of individual layers are recorded on a specific number of bites in fixed-point notation. Parameter to be tested was the correctness of classification, considered as ratio of the number of correctly classified cases to general number of cases.

Figures 4 and 5 illustrate research results for designed classifiers with vectors being recorded on specified number of bits. For learning data, the best results were achieved by LVQ neural network, CP is ranked second, and radial neural network is least suitable. The classifiers required seven, nine and eleven bits for vector recording, respectively, for the classification correctness be stabilized. Classification correctness for all networks equals $100 \%$. The same ranking persists for testing data analysis. The only difference is that classifiers required six, nine and ten bits for vector recording. Classification correctness for LVQ and CP networks oscillated around 90\%, whereas it was $80 \%$ for radial network.

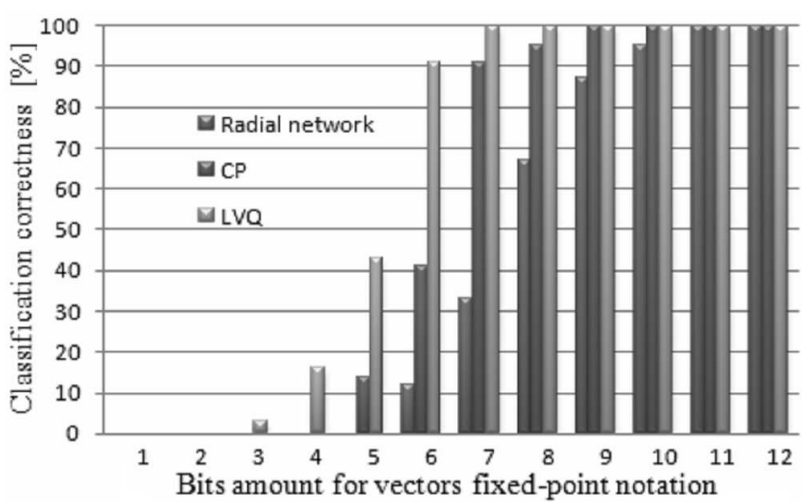

Fig. 4. Neural network operating correctness in relation to the number of bits assigned for fixed-point vector recording. Analysis done for teaching data.

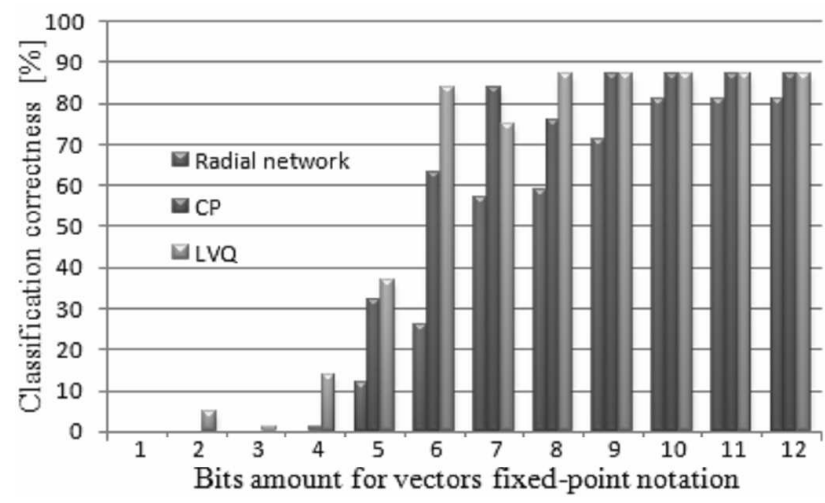

Fig. 5. Neural network operating correctness in relation to the number of bits assigned for fixed-point vector recording. Analysis done for testing data.

\section{Summary}

The research conducted enables to select the best architecture of specialist neural classifier in implementation for FPGA programmable devices. In executed analysis the best results were obtained by classifier based on LVQ artificial neural network, next was the classifier based on CP neural network, and then came, with lesser results, the classifier based on radial network. Eleven-bit recording format for input vectors and weight vectors was sufficient for correct operation of all classifiers under consideration.

\section{Acknowledgments}

This study is a part of the research project N R03 006106 supported by The National Centre for Research and Development NCBiR.

\section{References}

[1] C. Cempel, Vibroacoustics Diagnostics, Państwowe Wydawnictwo Naukowe, Warszawa 1989, p. 23 (in Polish). 
[2] W. Cioch, Machine Dynamics Problems 27, 57 (2003).

[3] S. Osowski, Neural Networks for Computing, Oficyna Wydawnicza Politechniki Warszawskiej, Warszawa 2000, p. 37 (in Polish).

[4] R. Tadeusiewicz, Neural Networks, Akademicka Oficyna Wydaw. RM, Warszawa 1993, p. 65 (in Polish).

[5] J. Majewski, P. Zbysiński, FPGA Devices in Examples, 1st ed., Wydawnictwo BTC, Warszawa 2007, p. 7 (in Polish).

[6] K. Skahill, Projecting of Programmable Logic Devices, 2nd ed., Wydawnictwo Naukowo-Techniczne, Warszawa 2004, p. 20 (in Polish).

[7] B. Łazarz, G. Wojnar, P. Czech, in: Maintenance Reliability 49, 68 (2011)

[8] B. Łazarz, P. Czech, A. Wilk, in: Second World Congress on Engineering Asset Management and the Fourth Int. Conf. on Condition Monitoring "WCEAM CM 2007”, Harrogate (UK) 2007, p. 1190.

[9] B. Łazarz, G. Wojnar, H. Madej, P. Czech, Mechanika (Lituania) 80, 56 (2009).
[10] H. Sarimveis, P. Doganis, A. Alexandridis, Adv. Eng. Software 37, 218 (2006).

[11] J. Adamczyk, W. Cioch, P. Krzyworzeka, Zagadnienia Eksploatacji Maszyn 34, 373 (1999).

[12] B. Samanta, Eng. Appl. Artificial Intellig. 16, 657 (2003).

[13] J. Żurada, M. Barski, W. Jędruch, Artificial Neural Networks, Wydawnictwo Naukowe PWN, Warszawa, 1996, p. 240 (in Polish).

[14] G.P. Zhang, IEEE Appl. Rev. 30, 451 (2000).

[15] T. Kohonen, Neurocomputing 21, 1 (1998).

[16] T. Masters, Neural Networks in Practice, Wydawnictwo Naukowo-Techniczne, Warszawa 1996, p. 15 (in Polish). 